\title{
Eosinophilic gastroenteritis presenting as small bowel obstruction: A case report and review of the literature
}

\author{
Min Young Yun, Young Up Cho, In Suh Park, Sun Keun Choi, Sei Joong Kim, Seok Hwan Shin, Kyung Rae Kim
}

Min Young Yun, Young Up Cho, Sun Keun Choi, Sei Joong Kim, Seok Hwan Shin, Kyung Rae Kim, Department of Surgery, College of Medicine, Inha University, Incheon, Korea

In Suh Park, Department of Pathology, College of Medicine, Inha University, Incheon, Korea

Correspondence to: Dr. Sei Joong Kim, Department of Surgery, Collage of Medicine, Inha University Shinheung-dong 3-ga, Junggu, 7-206, Incheon 400-103, Korea. ksj1511@inha.ac.kr Telephone: +82-32-8903431 Fax: +82-32-8903097

Received: 2006-11-08 Accepted: 2007-01-23

\begin{abstract}
Eosinophilic gastroenteritis is a rare disease of unknown etiology. It is characterized by eosinophilic infiltration of the bowel wall to a variable depth and symptoms associated with gastrointestinal tract. Recently, the authors experienced a case of eosinophilic gastroenteritis presenting as small bowel obstruction. A 51-year old woman was admitted to our hospital complaining of abdominal pain and vomiting. Physical examination revealed a distended abdomen with diffuse tenderness. Complete blood count showed mild leukocytosis without eosinophilia. Computed tomography confirmed a dilatation of the small intestine with ascites. An emergency laparotomy was performed for a diagnosis of peritonitis due to intestinal obstruction. Segmental resection of the ileum and end to end anastomosis were performed. Histologically, there was a dense infiltration of eosinophils throughout the entire thickness of ileal wall and eosinophilic enteritis was diagnosed. The patient recovered well, and was free from gastrointestinal symptoms at the time when we reported her disease.
\end{abstract}

(C) 2007 The WJG Press. All rights reserved.

Key words: Eosinophilic gastroenteritis; Small bowel obstruction; Eosinophilic infiltration

Yun MY, Cho YU, Park IS, Choi SK, Kim SJ, Shin SH, Kim $\mathrm{KR}$. Eosinophilic gastroenteritis presenting as small bowel obstruction: A case report and review of the literature. World J Gastroenterol 2007; 13(11): 1758-1760

http://www.wjgnet.com/1007-9327/13/1758.asp

\section{INTRODUCTION}

Eosinophillic gastroenteritis is an extremely rare disease, involving the entire gastrointestinal tract. It is characterized by diffuse eosinophilic infiltration in submucosal layer of the gastrointestinal tract $t^{[1-4]}$. The pathogenesis and etiology of eosinophilic gastroenteritis remain unclear ${ }^{[5-9]}$. Diagnostic criteria include demonstration of eosinophilic infiltration in the bowel wall, lack of evidence of extraintestinal disease, and exclusion of various disorders that mimic a similar condition ${ }^{[3]}$. We present a case of eosinophilic gastroenteritis that underwent emergency laparotomy for acute intestinal obstruction due to eosinophilic enteritis of the ileum.

\section{CASE REPORT}

A 51-year old woman presented to the emergency department with complaints of acute-onset of abdominal pain and vomiting. She did not receive any abdominal operation. The patient denied taking any drugs or herbal medicines. She had no history of drug allergy, asthma, or allergic rhinitis. Physical examination revealed that her abdomen was slightly distended and rigid with tenderness. Laboratory investigation showed a white cell count of $10800 / \mathrm{mm}^{3}$ with $3 \%$ eosinophils. Other laboratory studies were within normal limit. Abdominal radiograph displayed multiple air-fluid levels in the small intestine (Figure 1). Computed tomography demonstrated dilatation of the small intestine and ascites (Figure 2). With her abdominal distension and tenderness progressively worsening, emergency laparotomy was performed with a midline incision. Exploration revealed a large amount of yellowish ascites in the peritoneal cavity and focal stricture in the ileum about $60 \mathrm{~cm}$ proximal to the ileocecal valve. The remaining small bowel was dilated. Further inspection of the peritoneal cavity did not find any other abnormality. The stricture site was resected about $10 \mathrm{~cm}$ long and end to end anastomosis was performed. The mucosa of the specimen was edematous but there was no inflammation (Figure 3). Histologically, there was a dense infiltration of eosinophils throughout the entire thickness of ileal wall (Figure 4). Postoperatively, the patient's stool was negative for ova and parasites and skin prick test was negative. During the follow-up period of 1 year, the patient was completely free of recurrence.

\section{DISCUSSION}

Primary eosinophilic gastroenteritis is defined as a disorder that primarily affects the gastrointestinal tract with eosinophil-rich inflammation in the absence of known causes for eosnophilia, including drug reaction, 


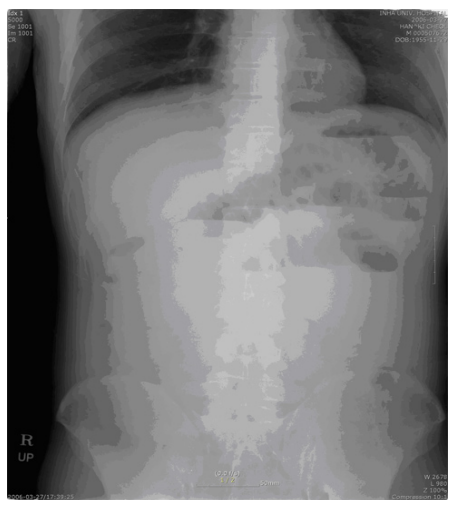

Figure 1 Erect abdominal X-ray showing multiple air-fluid levels in the small intestine.

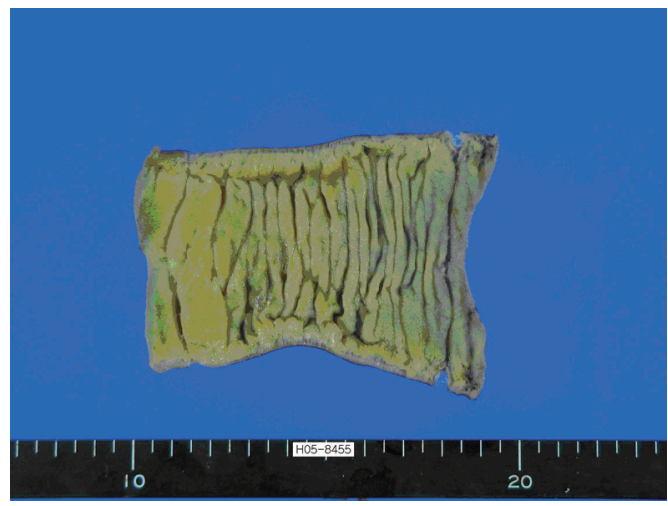

Figure 3 Resected specimen showing mucosa thickening.

parasitic infections, and malignancy ${ }^{[10]}$. Eosinophililc gastroenteritis was first described in 1937. In 1970, Klein classified the disease according to the predominance of eosinophilic infiltration in different layers of the intestinal wall $^{[11]}$. Involvement of different layers of the intestinal wall usually gives rise to different clinical manifestations. The mucosal form of eosinophilic gastroenteritis (most common variant) is characterized by vomiting, abdominal pain, diarrhea, blood loss in stools, iron-deficiency anemia, malabsorption, and protein-losing enteropathy. The muscularis form is characterized by infiltration of eosinophils predominantly in the muscle layer, leading to thickening of the bowel wall, which might result in gastrointestinal obstructive symptoms. The serosal form occurs in a minority of patients with eosinophilic gastroenteritis, and is characterized by exudative ascites with higher peripheral eosinophil counts compared with the other forms ${ }^{[3,10]}$. In our case, eosinophils infiltrated all three layers of the intestinal wall. Thickening of the muscle layers narrowed the intestinal lumen, causing intestinal obstruction, and serosal involvement produced ascites without peripheral eosinophilia.

Although eosinophilic gastroenteritis can involve any part of the gastrointestinal tract, the stomach and duodenum are the most common sites of involvement ${ }^{[2,3,6-8]}$. Because the pathogenesis and etiology of the disease are not well understood, no standards for the diagnosis of eosinophilic gastroenteritis exist. Talley $e t a l^{[12]}$ have identified three main diagnostic criteria: (1) the presence of gastrointestinal symptoms, (2) biopsies demonstrating eosinophilic infiltration of one or more areas of the

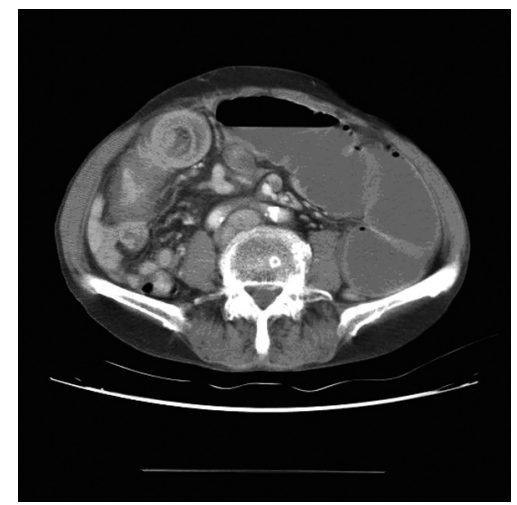

Figure 2 Computed tomography showing dilatation of the small intestine.

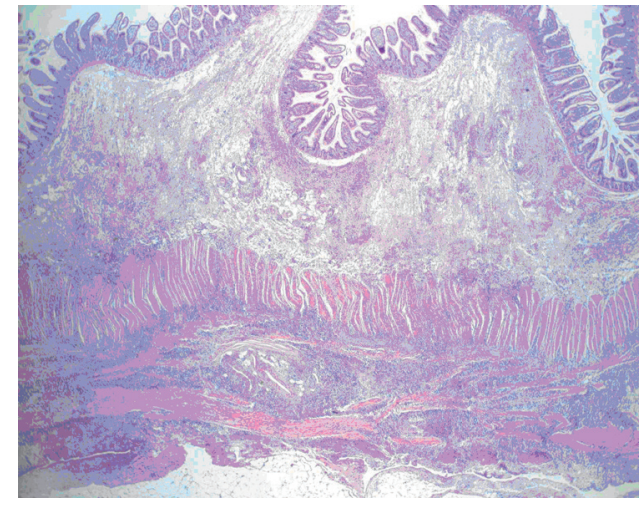

Figure 4 Microscopy of the resected ileum revealing eosinophilic infiltration from the submucosa to the subserosa (HE $\times 40)$.

gastrointestinal track, and (3) no evidence of parasitic or extraintestinal disease. It was reported that peripheral eosinophilia is uniformly associated with eosinophilic gastroenteritis ${ }^{[6,7]}$. However, the definitive diagnosis of eosinophilic gastroenteritis requires histologic evidence of eosinophilic infiltration ${ }^{[2]}$. When subserosal disease involves the small bowel, biopsy of the mucosal layer taken during gastroscopy or colonoscopy often fails to diagnose eosinophilic gastroenteritis. Laparotomy or laparoscopic full thickness biopsy is often required to make a diagnosis in such case.

Treatment with a steroid is the mainstay in the management of eosinophilic gastroenteritis. Although surgeons should avoid unnecessary surgery, surgical intervention may sometimes be required when a definitive diagnosis cannot be made or when obstruction or perforation occurs, as in this patient.

Because the natural history of eosionphilic gastroenteritis has not been well documented, long-term follow-up is required. Moreover, these diseases can often be a manifestation of another primary disease process, routine surveillance of the cardiopulmonary systems is recommended ${ }^{[11]}$.

\section{REFERENCES}

1 Chang IS, Yoo SY, Kim KK, Kim SY. Eosinophilic enteritis of the cecum. J Korean Surg Soc 1984; 26: 40-45

2 Chen MJ, Chu CH, Lin SC, Shih SC, Wang TE. Eosinophilic gastroenteritis: clinical experience with 15 patients. World J Gastroenterol 2003; 9: 2813-2816 
3 Hsu YQ, Lo CY. A case of eosinophilic gastroenteritis. Hong Kong Med J 1998; 4: 226-228

4 Biswas S, Hoo W, Katsoulas N, Munro J, Oke O. Eosinophilic enteritis: a rare cause of abdominal pain. Int J Colorectal Dis 2007; 22: 87-88

5 Whitaker IS, Gulati A, McDaid JO, Bugajska-Carr U, Arends MJ. Eosinophilic gastroenteritis presenting as obstructive jaundice. Eur J Gastroenterol Hepatol 2004; 16: 407-409

6 Caldwell JH. Eosinophilic Gastroenteritis. Curr Treat Options Gastroenterol 2002; 5: 9-16

7 Alexander $\mathbf{P}$, Jacob S, Paul V. Laparoscopy in eosinophilic jejunitis presenting as subacute bowel obstruction: a case report. Trop Gastroenterol 2003; 24: 97-98

8 Alamo Martínez JM, Ibáñez Delgado F, Galindo Galindo A, Bernnal Bellido C, Durén Ferreras I, Suárez Artacho G, Martínez Vieira A. Intestinal obstruction by eosinophilic jejunitis. Rev Esp Enferm Dig 2004; 96: 279-283

9 Charalabopoulos A, Charalabopoulos K, Avuzuklidou M, Charalabopoulos AK, Ioachim E, Lebren F, Fotiadis K. Eosinophilic gastroenteritis: presentation of two patients with unusual affect of terminal ileum and caecum with manifestations of acute abdomen and literature review. Int J Clin Pract 2004; 58: 413-416

10 Rothenberg ME. Eosinophilic gastrointestinal disorders (EGID). J Allergy Clin Immunol 2004; 113: 11-28; quiz 29

11 Klein NC, Hargrove RL, Sleisenger MH, Jeffries GH. Eosinophilic gastroenteritis. Medicine (Baltimore) 1970; 49: 299-319

12 Talley NJ, Shorter RG, Phillips SF, Zinsmeister AR. Eosinophilic gastroenteritis: a clinicopathological study of patients with disease of the mucosa, muscle layer, and subserosal tissues. Gut 1990; 31: 54-58

S-Editor Wang J L-Editor Wang XL E- Editor Zhou T 